\title{
Im Gedenken der Kinder. Die Kinderärzte und die Verbrechen an Kindern in der NS-Zeit
}

Herausgegeben von der Deutschen Gesellschaft für Kinder- und Jugendmedizin (DGKJ) Begleitheft zur Gedenkveranstaltung und Ausstellung der DGKJ

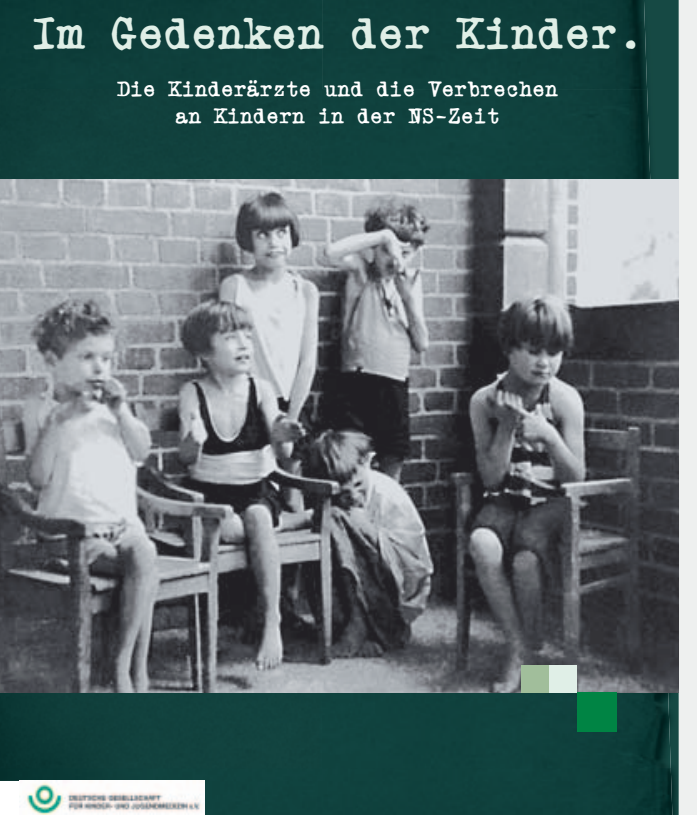

굴 $=$
2 Vorwort

3 Editorial F. Zepp

4 Erklärung der Deutschen Gesellschaft für Kinderund Jugendmedizin e.V. (DGKJ) anlässlich der Gedenkveranstaltung am 18. September 2010 in Potsdam

6 "Was habt ihr mit meinem Kind gemacht, das ist ja mein Kind gar nicht mehr! ..." - Zeitzeugnisse

9 Der Nationalsozialismus als biopolitische Entwicklungsdiktatur Konsequenzen für die Kinderheilkunde H.-W. Schmuhl

13 Im Gedenken der Kinder. Die Kinderärzte und die Verbrechen an Kindern in der NS-Zeit - Ausgewählte Tafeln aus der Ausstellung in Potsdam-Babelsberg, 16.09.-19.09.2010

21 Vorschau

21 Impressum
Bildnachweis: Alle Bilder @ Institut für Geschichte der Medizin,

PD Dr. Thomas Beddies, Charité-Universitätsmedizin Berlin,

Zentrum für Human- und Gesundheitswissenschaften 


\section{Im Gedenken der Kinder}



Im vorliegenden Supplement werden Texte und Bilder publiziert, die im Rahmen der Ausstellung und Gedenkveranstaltung "Im Gedenken der Kinder. Die Kinderärzte und die Verbrechen an Kindern in der NS-Zeit" verlesen und gezeigt wurden.

Die DGKJ bedankt sich bei allen, die die Konzeption und die Organisation dieser Veranstaltungen unterstützt haben. Hier sei vor allem die "Arbeitsgruppe Potsdam 2010" genannt:

- PD Dr. Thomas Beddies

- Dr. Petra Fuchs

- Prof. Dr. Gerhard Gaedicke

- Dr. Kristina Hübener

- Dr. Astrid Ley

- Dr. Gudrun Noleppa

- Prof. Dr. Lothar Pelz

- Prof. Dr. Michael Radke

- Wolfgang Rose, MA

- Werbefotografie Wolfgang Chodan
Ebenso gilt unser Dank Prof. Dr. Hans-WalterSchmuhl für seinen Vortrag "Der Nationalsozialismus als biopolitische Entwicklungsdiktatur Konsequenzen für die Kinderheilkunde", Nadja Uhl, die in beeindruckender Weise aus Originalbriefen gelesen hat, sowie Mitgliedern des "Orchesters der Deutschen Kinderärzte" für die musikalische Umrahmung der Gedenkveranstaltung.

An dieser Stelle möchten wir auch dem SpringerVerlag und der Redaktion der "Monatsschrift Kinderheilkunde", die die Erstellung des Supplements für die DGKJ ermöglicht haben, ganz herzlich unseren Dank aussprechen.

Der Vorstand der DGKJ

Berlin, Januar 2011

Kontaktadresse:

Deutsche Gesellschaft für Kinder-

und Jugendmedizin (DGKJ)

Chausseestr. 128/129, 10115 Berlin

info@dgkj.de

\section{Freier Online-Zugang}

zu dem Supplement „Im Gedenken der Kinder" der Deutschen Gesellschaft für Kinder- und Jugendmedizin

Greifen Sie in unserem

Onlinearchiv kostenlos auf alle Beiträge

dieses Supplements zu. 


\section{Editorial}

\section{Liebe Leserinnen und Leser, liebe Kolleginnen und Kollegen,}

dieses Sonderheft fasst Ergebnisse einer langjährigen kritischen Auseinandersetzung der Deutschen Gesellschaft für Kinder- und Jugendmedizin (DGKJ) mit der Verwicklung der Kinderheilkunde und ihrer Repräsentanten in die Verbrechen der Kinder-„,Euthanasie“ in der NS-Zeit zusammen.

Schon seit vielen Jahren befasst sich die DGKJ mit der Aufarbeitung ihrer Geschichte während der Zeit des Nationalsozialismus. Die 1984 gegründete Historische Kommission hat diese Aufgabe mit großer Ernsthaftigkeit vorangetrieben, entscheidende Beiträge geleistet und wichtige Impulse gegeben. Bereits 1998 hat sich die DGKJ in der „Dresdner Erklärung “ mit der Gleichschaltung der Kinderheilkunde im Nationalsozialismus befasst. Dabei ging es um das Schicksal hunderter jüdischer Kinderärzte zwischen 1933 und 1945, die auch unter Mitwirkung ihrer Kolleginnen und Kollegen ausgegrenzt und vertrieben wurden. Die persönlichen Schicksale, die häufig in den NS-Konzentrationslagern endeten, wurden nachgezeichnet und dokumentiert. $^{2}$

Anlass und Auftrag für die DGKJ, sich erneut mit der Rolle der Kinderheilkunde im Nationalsozialismus zu befassen, ist das Gedenken an die ab 1940 aufgebauten sogenannten „Kinderfachabteilungen“

\footnotetext{
1 Monatsschrift Kinderheilkunde, Mai 1999, 147. Band, Suppl. 1.

2 Eduard Seidler: Jüdische Kinderärzte 1933-1945. Entrechtet - Geflohen - Ermordet. Erweiterte Neuauflage Basel/Freiburg 2007.
}

zur Ermordung kranker und behinderter Kinder. Mehr als 10.00o Kinder und Jugendliche fielen bis 1945 den Programmen zur Vernichtung sogenannten „lebensunwerten Lebens“ zum Opfer. In vielen Fällen waren es Kinderärzte und Kinderärztinnen, die die Kinder im Namen der Wissenschaft meldeten, begutachteten, in Experimenten benutzten und töteten. Diese Verbrechen fanden im Zentrum des gesellschaftlichen Lebens in Deutschland statt, in Arztpraxen und Krankenhäusern, staatlichen Ämtern und wissenschaftlichen Instituten.

Im September 2010 veranstaltete die DGKJ auf ihrer Jahrestagung in Potsdam eine zentrale Gedenkveranstaltung zur Erinnerung an die Opfer der NS-Medizinverbrechen an Kindern. Vor den 800 Teilnehmern der Gedenkfeier wurde eine Erklärung verlesen, die die Anerkennung von Schuld und die Mitwirkung von Kinderärzten und -ärztinnen an den NSMedizinverbrechen festhält (s. S. 4 f.).

Die Erklärung der DGKJ bekräftigt auch die Selbstverpflichtung der Wissenschaft, ihre Grenzen in den unverzichtbaren Rechten und der unantastbaren Würde jedes einzelnen Menschen zu sehen. Die DGKJ appelliert zudem an alle Forscher und Wissenschaftler, sich jedweder Form von Mitläufertum und Meinungskonformismus entgegenzustellen.

Der Frage, wie und warum Kinderärztinnen und -ärzte zu Tätern der NS-Medizinverbrechen wurden, wie sie ihr Handeln rechtfertigten und nach 1945 unangetastet weiter praktizierten, bewegt wohl alle, die sich mit diesem grauenvollen und bedrückenden Thema befasst haben. Hans-Walter Schmuhl geht in seinem Beitrag „Der Nationalsozialismus als biopolitische Entwicklungsdiktatur" (S. 9 ff.) dem Werdegang einiger prominenter Kinderärzte nach.

Die Briefe von Eltern, deren Kinder in „Kinderfachabteilungen“ untergebracht wurden (S. 6 f.), sprechen von Sorge und Trauer, teils von Misstrauen gegenüber den Institutionen, aber auch von offener Dankbarkeit für die Tötung des Kindes, das als Belastung für Familie und Volk empfunden wurde.

Die Abbildungen und Fotos, die dieses Sonderheft begleiten, entstammen der Ausstellung „Im Gedenken der Kinder. Die Kinderärzte und die Verbrechen an Kindern in der NS-Zeit“. Die Bildtafeln stellen die politischen, wirtschaftlichen und sozialen Dimensionen der NS-Medizinverbrechen dar, sie geben aber auch berührende Einblicke in das kurze Leben von Kindern, die der Gesellschaft „lebensunwert" waren.

Diese Vergangenheit der Kinderheilkunde in Deutschland wird niemals „Geschichte sein“ im Sinne eines abgeschlossenen, düsteren Kapitels der Historie - die Opfer dieser Verbrechen dürfen nie vergessen werden.

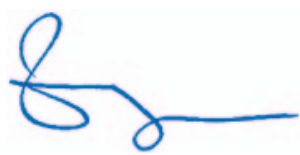

Prof. Dr. Fred Zepp

Präsident der DGKJ 


\title{
Im Gedenken der Kinder. Die Kinderärzte und die Verbrechen an Kindern in der NS-Zeit
}

\author{
Erklärung der Deutschen Gesellschaft für Kinder- und Jugendmedizin e.V. (DGKJ) \\ anlässlich der Gedenkveranstaltung am 18. September 2010 in Potsdam
}

\section{Meine Damen und Herren,}

im Jahr 2008 feierte die Deutsche Gesellschaft für Kinder- und Jugendmedizin ihr 125-jähriges Bestehen. Unsere wissenschaftliche Fachgesellschaft hat gegenwärtig über 14.00o Mitglieder, mehr als die Hälfte von ihnen sind jünger als 45 Jahre, also mehr als 20 Jahre nach dem Ende des Zweiten Weltkriegs geboren.

Vielleicht liegt in dieser Altersstruktur ein Grund dafür, dass - nach einer langen Phase kollektiver Verdrängung - in der zurückliegenden Dekade auf den Jahrestagungen unserer Gesellschaft gleich mehrere gut besuchte und erfolgreiche wissenschaftliche Symposien zum Thema „Kinderheilkunde und Nationalsozialismus“ stattfinden konnten. Und besonders die 1998 in Dresden durchgeführte Gedenkveranstaltung für rassisch und politisch verfolgte Pädiater in der NS-Zeit kann als markantes Ereignis in einem noch andauernden Prozess der Bewältigung des „Nationalsozialismus in der Kinderheilkunde“ verstanden werden: Der Auftrag, eine Dokumentation möglichst aller nach 1933 verfolgten, vertriebenen und ermordeten kinderärztlichen Kolleginnen und Kollegen zu erarbeiten, erging seinerzeit vom Vorstand und von der Mitgliederversammlung der Deutschen Gesellschaft für Kinder- und Jugendmedizin. Die publizierte Dokumentation konnte inzwischen in erweiterter Form neu aufgelegt werden ${ }^{1}$ und

1 Eduard Seidler: Jüdische Kinderärzte 1933-1945. Entrechtet - Geflohen - Ermordet. Erweiterte Neuauflage Basel/Freiburg 2007. hat international höchstes Lob und Würdigung erfahren.

In Anerkennung der Tatsache, dass lange - zu lange - nichts zur Aufklärung der Rolle der Kinderärzte in der NS-Zeit unternommen wurde und dass auch unsere Fachgesellschaft sich erst spät ihrer Verantwortung in dieser Frage gestellt hat, unternehmen wir jetzt einen zweiten bedeutsamen und schwierigen Schritt zur Aufarbeitung der Geschichte der deutschen Kinderheilkunde in den Jahren zwischen 1933 und 1945.

Wir gedenken der minderjährigen Opfer des menschenverachtenden Programms von „Auslese und Ausmerze“ der NS-Medizin, an dem auch Kinderärztinnen und Kinderärzte beteiligt waren.

Wir gedenken der Kinder und Jugendlichen, die aus der so genannten Volksgemeinschaft ausgegrenzt, asyliert und sterilisiert wurden, die für Menschenversuche missbraucht und zu Tausenden in die Kranken-Mordaktionen des Zweiten Weltkriegs einbezogen wurden. Es handelte sich um kranke und behinderte Jungen und Mädchen, die von Ärzten und Erziehern nicht als Patienten oder als schutzbefohlene Heimzöglinge behandelt wurden, sondern für fragwürdige Experimente missbraucht, deportiert und umgebracht wurden. Dabei wurde gleichzeitig die allzeit bestehende Abhängigkeit des Kindes vom Erwachsenen, also der Vertrauensvorschuss, den das Kind zu gewähren gezwungen ist, durch Ärzte und Pfleger in gröbster Weise verraten. Auch die Eltern und Angehörigen wurden vor- sätzlich getäuscht: Sie gaben ihre Kinder häufig in der Hoffnung auf eine moderne, Erfolg versprechende Behandlung in die Hände der Täter. Mehr als 10.0oo Kinder und Jugendliche wurden bis zum Ende des Zweiten Weltkriegs Opfer der verschiedenen Programme zur Vernichtung sog. „lebensunwerten Lebens"; viele von ihnen wurden von Kinderärzten an politisch indoktrinierte, staatliche Gesundheitsbehörden gemeldet, begutachtet, für Experimente herangezogen und getötet. (Anm.: Sie finden diese Tatsachen in der Ausstellung „Im Gedenken der Kinder“, die hier ab S. 13 dokumentiert ist.) Die in den zurückliegenden Jahren erarbeiteten wissenschaftlichen Befunde konfrontieren uns mit der Tatsache, dass auch Kinderärzte die körperliche Unversehrtheit ihrer Patienten missachtet und sie für politisch motivierte Interessen missbraucht haben. Es waren Ärzte, die über die ihnen anvertrauten Kinder die Todesurteile fällten, ihnen tödliche Dosen von Luminal verabreichten. Es waren Ärzte, die zusahen, wie viele Kinder in den „Kinderfachabteilungen“ verhungerten. Rechtliche Konsequenzen hatten sie in dieser Zeit nicht zu fürchten.

Wir bekennen die geistige Miturheberschaft und das aktive Mittun von Kinderärztinnen und Kinderärzten an diesen Verbrechen; wir beklagen darüber hinaus jede Form von Mitläufertum und Meinungskonformismus, ohne die das Regime nicht hätte funktionieren können und die es den Tätern erst möglich machte, ihre Verbrechen durchzuführen. Verbrechen, die ja nicht im Ungewissen ferner Landstriche 
und besetzter Gebiete, sondern in der Mitte des gesellschaftlichen Lebens und mitten in Deutschland in Arztpraxen und Krankenhäusern, staatlichen Ämtern und wissenschaftlichen Instituten stattfanden.

Zweifellos ist es schmerzlich, geschichtliche Tatsachen rückhaltlos offen zu legen und Licht in das Dunkel der eigenen Vergangenheit zu bringen. Die Wahrheit anzunehmen, Schuld zu bekennen und sich dieser Verantwortung zu stellen, ist aber gleichzeitig, so hoffen wir, eine angemessene Art der Entschuldigung bei den Opfern und ihren Angehörigen, von denen nicht wenige noch heute unter den Folgen der historischen Ereignisse leiden.

Wir können dieses Leid nicht löschen, aber wir können uns dessen bewusst werden und dürfen nicht aufhören, uns mit der Vergangenheit zu beschäftigen und nach Wegen der Aufarbeitung zu suchen. Erste Schritte sind mit Beginn der systematischen Bearbeitung der NS-Vergangenheit getan, und so stellt sich die Deutsche Gesellschaft für Kinder- und Jugendmedizin dieser Aufgabe.

Wir müssen uns vor allem dafür einsetzen, dass Ausgrenzung, Angriffe auf die Menschenwürde und menschenverachtende Ideologien in unserer Gesellschaft keine Chance mehr erhalten.

Meine Damen, meine Herren!

Jeder Wissenschaft und insbesondere den biowissenschaftlichen und medizinischen Disziplinen gehören klare ethische und rechtliche Grenzen gesetzt. Es darf keine Zielvorgaben in der Forschung und in der Betreuung von Patienten geben, die als so wichtig und hochrangig angesehen werden können, dass sie die Missachtung individueller Menschenwürde und Menschenrechte rechtfertigen. Wissenschaft findet ihre Grenzen in den unverzichtbaren Rechten und der unantastbaren Würde jedes einzelnen Menschen. Davon sind Kinder und Jugendliche nicht ausgenommen.

Der Nationalsozialismus hatte Ärzte und Forscher weitgehend von ihrer Verantwortung gegenüber ihren Patienten entbunden, indem er scheinbar höhere Werte und politische Interessen des Volksganzen in den Vordergrund stellte. Wir haben uns der Tatsache zu stellen, dass viele Kinderärztinnen und Kinderärzte nicht die Kraft aufbrachten, den Versuchungen zu widerstehen. Sie haben damit sich und der deutschen Kinderheilkunde schwerste Schuld aufgeladen.

Wir verneigen uns heute in Demut vor den Opfern und ihren Angehörigen und bitten im Namen der Deutschen Gesellschaft für Kinder- und Jugendmedizin um Verzeihung für das Leid, das Kinderärztinnen und Kinderärzte ihnen in dieser Zeit zugefügt haben.

Potsdam, 18. September 2010

Prof. Dr. Fred Zepp

Präsident der Deutschen Gesellschaft

für Kinder- und Jugendmedizin (DGKJ) 


\section{„Was habt ihr mit meinem Kind gemacht, das ist ja mein Kind gar nicht mehr! ..." - Zeitzeugnisse}

\begin{abstract}
Bei den nachfolgend abgedruckten Dokumenten handelt es sich ganz überwiegend um Briefe von Eltern, deren Kinder in die Maßnahmen zur Vernichtung "lebensunwerten" Lebens einbezogen wurden oder einbezogen werden sollten.

Das Spektrum der Reaktionen reicht von unverhohlener Zustimmung über resignierende Hinnahme bis zur bitteren Klage über die Behandlung und Ermordung der Kinder.

Selbstverständlich verbietet sich aus der ausschließlichen Lektüre der hier isoliert wiedergegebenen Texte eine wertende Beurteilung im Einzelfall. Spürbar werden aber in vielen Briefen wie auch in der zusammenfassenden Betrachtung der Dokumente, die Nöte und Schrecknisse, denen man die Angehörigen mit der Zumutung der Preisgabe ihrer Kinder aussetzte.
\end{abstract}

\section{Brief 1}

Eine Pflegemutter aus Mannheim schreibt im Oktober 1940 an den Leiter der „Erziehungs- und Pflegeanstalt für Geistesschwache Mosbach/Schwarzacher Hof":

„Geehrter Herr Direktor!

Ihre so herzliche Teilnahme an dem Heimgang meiner lieben Elfriede habe ich dankend erhalten. Todesursache angeblich Krampf, aber ich bin anderer Meinung, das Gesetz, das vor Jahren geplant, ist zur Ausführung gekommen.

Die armen kranken Menschen, wie das Vieh zum Schlachthof geführt (...). Das ist ja ein Massensterben, fällt doch auf, ist hier allgemeines Stadtgespräch. Kenne vier Kranke, wo auch so plötzlich gestorben sind (...). Der alte Gott, er lebt noch. Bitte Herrn Direktor (um) Diskussion übermein Schreiben, bin so aufgeregt.

Herzliche Grüße nebst Pflegerinnen“

\section{Brief 2}

Eine Mutter, die ihr Kind in der Psychiatrischen und Nervenklinik der Berliner Charité vorgestellt hatte, schreibt im November 1941 an die dortige Fürsorgerin:

„Liebes Fräulein Pederzani!

Nun sind wir schon wieder eine Woche zu Hause, wie schnell doch die Zeit vergeht bei diesen kurzen Tagen. Die Reise ist ganz gut verlaufen und die Zahnwunden bei unserem Rolf sind schon gut verheilt.

Ich kann es noch immer gar nicht fassen, dass er sich nun so weiter abquälen muß in seinem Leben, und weil er trotzallem so ein sonniges Gemüt hat, werde ich es wohl nie übers Herz bringen, ihn in eine Anstalt zu tun.

Hoffentlich erleben wir es mal noch, dass eine Ärzte-Kommission gegründet wird, die über das Leben solcher Unheilbarkranken mit der Erlösung entscheidet, zum Segen der Kranken selbst und auch für die Angehörigen. (...) ich danke Ihnen und Ihren Helferinnen für Ihre Liebe und Güte, die sie uns entgegenbrachten.

Freundliche Grüße für alle auf Station 7 und Heil Hitler"
Brief 3

Der Direktor der Universitäts-Kinderklinik Heidelberg, Prof. Dr. Johann Duken, an einen Vater (Juli 1942):

"Heute morgen habe ich die Nachricht von Herrn Prof. Schneider erhalten, daß am Freitag Ihr Kind in eine andere Anstalt verlegt wird. (Anm.: „andere Anstalt“ war die Tarnbezeichnung für die Verlegung in eine Tötungsanstalt.)

Sie werden von dort aus näheren $\mathrm{Be}$ scheid erhalten. Ich möchte Ihnen in diesem Augenblick raten, Ihrer Gattin zunächst gar nichts zu sagen, damit sie dann einfach eines Tages vor der festen Tatsache steht. Sollten für Sie selbst irgendwelche Fragen auftauchen, so bitte ich Sie, mich anzurufen.

Ich benutze diese Gelegenheit (Ihnen) und ganz besonders Ihrer Gattin, von Herzen alles Gute für die Zukunft zu wünschen. Möchte der Kummer sich bald verziehen und dann der Raum für neues Werden frei werden!

Mit den besten Empfehlungen und Heil Hitler"

Brief 4

Ein Vater schreibt an den Chefarzt der städtischen Nervenklinik für Kinder in Berlin-Wittenau:

„Im Felde Nordfront, den 3. Dezember 1942. Betrifft: Rosemarie K. (...) Durch die Zeilen meiner Frau habe ich erfahren das mein Kind Rosemarie (...) vom RittbergHaus Bln.-Lichterfelde nun in Ihre Klinik überwiesen ist. Ich wende mich nun an Sie mit der Bitte mir ein ärztliches Attest über den Zustand meiner Tochter Rosemarie zu 
Eetunald, g4. 11. 41

Linon Ininlein Pederzani!







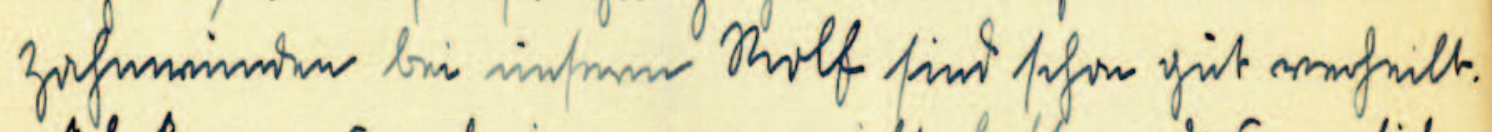



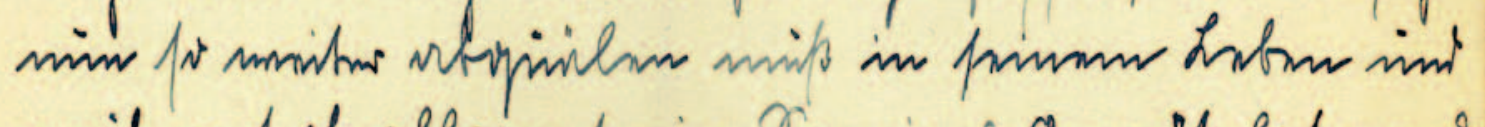

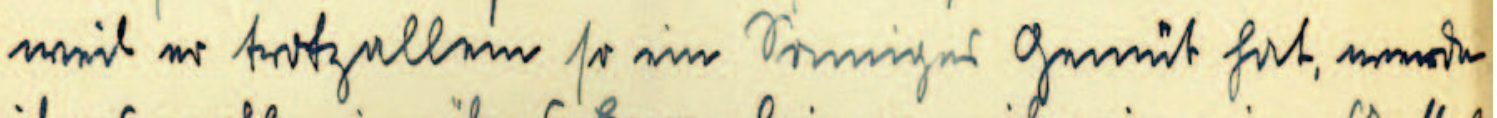



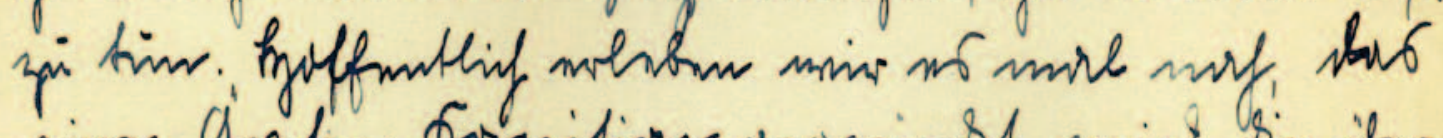

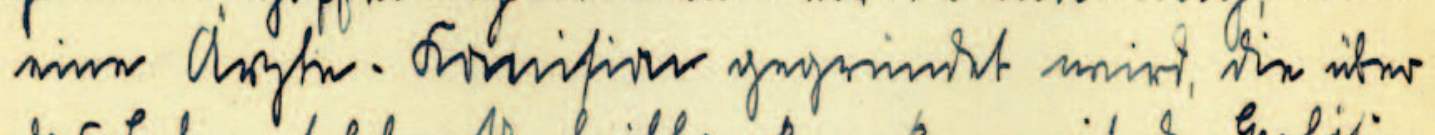

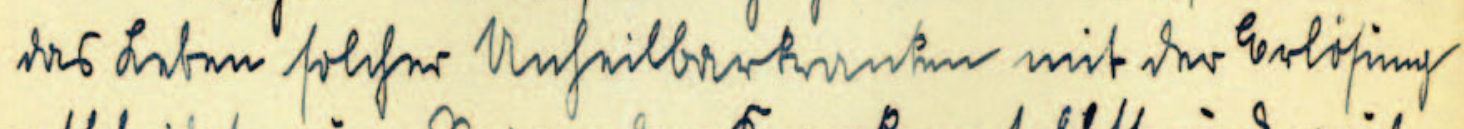



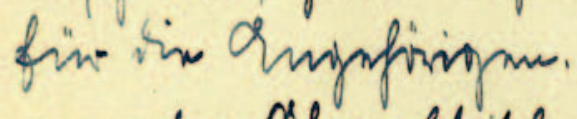





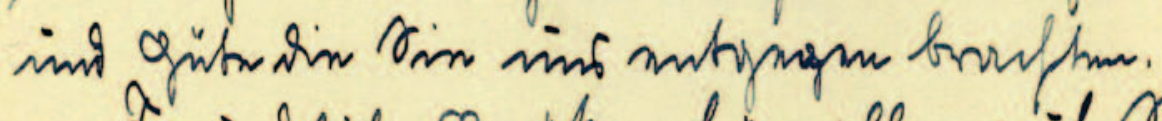

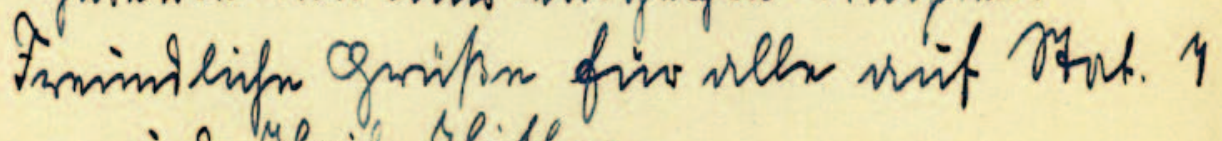
imm yeris tithw

Geruten. 1.

timpinte is

Brief 2: Originalbrief 29.11.1941 (Mit freundlicher Genehmigung des Instituts für Geschichte der Medizin, Berlin)

Monatsschrift Kinderheilkunde Supplement 1 - $2011 \mid 7$ 
senden. Bitte aber mir tatsächlich das mitzuteilen wie es nun auch für später um meine Tochter gestellt ist. Ich als Frontsoldat habe seit 1939 viel hinter mir und bin alles gewöhnt. Als SS Angehöriger kann ich mich nicht von dem Gedanken lösen, daß solch ein Kind dem Staat eine ungeheure Last ist und das Pflegepersonal jetzt, wo wir im totalen Krieg stehen, meinen verwundeten $\mathrm{Ka}$ meraden zu gute kommen kann. Bin selbst 2 mal verwundet und wei $\beta$, was eine Schwester für uns im Lazarett bedeutet.

Habe jetzt ein Gesuch an meinen Bataillons-Kommandeur geschrieben um Urlaub, um das alles, was mich als SS Mann beschäftigt, zu regeln und schließlich ein sanftes Entschlafen zu erwirken. Brauche dazu aber das verlangte Attest, um Unterlagen zwecks Urlaub zu haben. Auch will ich meiner Frau das alles leichter machen den Abschied vom Kinde.

Heil Hitler"

\section{Brief 5}

Brief eines Vaters, der als Soldat in Frankreich steht, an die Direktion der Landesheilanstalt Hadamar (März 1943):

„Von der Heil-und Pflegeanstalt Scheuern (...) habe ich Nachricht erhalten, daß mein Kind Willi in Ihre Anstalt verlegt wurde. Ein Päckchen mit Keks und Bonbons wird ihn nicht mehr lebend erreicht haben. Ich erhielt von meiner Frau die bittere Nachricht, daß mein Kind bereits zur letzten Ruhe gebettet ist.

So habe ich mir den Heimgang meiner Kinder nicht vorgestellt. Mein Kind verließ die Anstalt Scheuern bei Wohlbefinden. Innerhalb von 8 Tagen krank, tot, beerdigt, ohne daß ich eine Ahnung davon habe. Ich könnte es begreifen vor eine solche bittere Tatsache gestellt zu sein, bei meinem ältesten Sohne, als Soldat, er steht an der Ostfront, war schon 2 mal verwundet.

Ich stehe kurz vor meinem Urlaub und hätte auf eine Nachricht von Ihnen in diesem Falle, den Urlaub sofort antreten können. Es war meine Absicht das Kind heimzuholen. In der Anstalt Scheuern war ich mit der Unterbringung zufrieden. Bei Ihnen hätte ich mich erst überzeugen müssen. Ich kann mich nicht damit abfinden, mir kommt es vor, als wenn mein Kind behandelt worden wäre wie erblich belastet oder wie das Kind eines Trinkers. Ich verwahre mich dagegen. Im Privatleben unbeschol- ten. Als Soldat, einberufen 1914/18 von Anfang bis Ende, mit Auszeichnung, verwundet, Führung gut und unbestraft.

Seit 1. September 1939 stehe ich ebenfalls wieder für Volk und Führer unter Waffen.

Heil Hitler"

\section{Brief 6}

Eine Mutter an die Oberärztin der Heilund Pflegeanstalt Brandenburg-Görden vom November 1943:

„Sehr geehrte Frau Doktor Pusch!

Schrecklich beeindruckt hat mich der Besuch am letzten Mittwoch bei meinem Söhnchen Hans-Dieter (...). Tief erschüttert trat ich meinen Heimweg an und aus diesem Gefühl heraus und um die Sorge um mein Kind muß ich Ihnen heute schreiben und Sie bitten mir ganz offen und ehrlich zu sagen, wie es mit meinem Kind steht. Entsetzt war ich, wie sehr sich das Kind in verhältnismäßig kurzer Zeit verändert hat und verfallen war. Ich bin stark genug, die volle Wahrheit zu verstehen und zu tragen. Wenn Sie als Ärztin ja ihre Kranken auch mit anderen Augen sehen, so nehme ich an, daß sie aber auch mich als Mutter verstehen, die ihr Kind liebt und besonders wenn es so leiden muß. Ich vergesse nie die unsagbar traurigen Augen und das Gesichtchen wie ein kleiner Greis. (...) Es ist doch so für das Kind und auch für mich als Mutter, die ich schon recht viel seelische Kummer und Leid (mit)gemacht habe, eine Qual.

(...) Ich bitte Sie also nochmals herzlichst, mir baldmöglichst Nachricht zu geben, was sie von Dieters Krankheit halten, sind es nun nur die Versuche, die ihn jetzt so schnell abfallen ließen oder geht es mit ihm rapide $z$ En Ende? (...)“

\section{Brief 7}

Eine Mutter, deren Tochter in der Anstalt „Schwarzacher Hof “ lebte, schreibt im Juli 1944 an die Psychiatrische Universitätsklinik in Heidelberg:

„Es wurde mir bekannt, daß Erika in eine andere Anstalt verlegt werden soll. Was das heißt, weiß ich. Mein heißer Wunsch wäre, das arme Kind möchte seine Ruhe haben, daß es aber für eine Mutter sehr hart ist $z u$ wissen, auf welche Art es geschieht, dürfen Sie mir glauben. Könnte man das Kind nicht dort einschlafen lassen, wo seine zweite Heimat war und ich mir das Kind dann heimholen könnte? Muß es so sein, daß das
Kind dort wegholt wird und mir einfach die Asche zugeschickt wird? Gewiß es geschehen heute noch andere Dinge. Aber zu der Sorge um meinen Mann und der vielen Arbeit, die auf mir ruht, kommt dann noch dieser quälende Gedanke: Was geht jetzt mit dem Kinde vor, wo werden sie es hintun? Könnten Sie das Kind nicht nach Heidelberg in die Klinik holen und es dort einschlafen lassen?"

\section{Brief 8}

1984 berichtet eine Mutter im Rahmen eines Ermittlungsverfahrens von dem Wiedersehen mit ihrer damals siebenjährigen Tochter in der Psychiatrischen Universitätsklinik Heidelberg im Herbst 1944:

„Meine Tochter hat auf mich fast nicht reagiert. Ich war fassungslos, wie abgemagert und apathisch mein Kind war. Ich nahm meine Tochter in den Arm und heulte los. Ich rief: „Was habt Ihr mit meinem Kind gemacht, das ist ja mein Kind gar nicht mehr!" (...) Ich habe in meiner Erregung weiter geäußert: „Wer hat das Recht, mir das Kind zu nehmen - Ihr laßt es ja verhungern!“ (...).

Die Ordensschwester sagte zu mir, ich müsse schnell handeln und alles versuchen, daß ich das Kind wiederbekomme, ehe es zu spät sei.

Bei zwei weiteren Besuchen findet die Mutter das Kind in noch schlechterem Zustand vor. Schließlich kann sie die Entlassung durchsetzen:

Die Schwestern schienen mir bereits vorbereitet gewesen zu sein und auch froh, daß sie mir das Kind übergeben durften. (...) Ich hatte für Renate neue Kleider mitgebracht, die ihr auf der Station angezogen worden sind. Mir wurde Renate ins Besuchszimmer gebracht. Sie konnte nicht mehr laufen. Ich wickelte sie in eine Decke und trug sie aufden Armen aus der Klinik. (...) Ich habe meine Tochter zu Hause langsam wieder aufpäppeln müssen. Nach ein paar Wochen konnte sie wieder laufen und freudige Reaktionen zeigen.

Sie lebt heute in der Heil- und Pflegeanstalt Stetten."

\section{Zusammenstellung und Bearbeitung:}

\section{PD Dr. Thomas Beddies}

Charité - Universitätsmedizin Berlin Zentrum für Human- und Gesundheitswissenschaften Institut für Geschichte der Medizin Historische Kommission der DGKJ 


\title{
Der Nationalsozialismus als biopolitische Entwicklungs- diktatur - Konsequenzen für die Kinderheilkunde
}

\author{
Vortrag von apl. Prof. Dr. Hans-Walter Schmuhl anlässlich der Gedenkveranstaltung \\ der Deutschen Gesellschaft für Kinder- und Jugendmedizin e.V. (DGKJ) \\ am 18. September 2010 in Potsdam
}

Am 5. Januar 1944 schrieb Prof. Jussuf Ibrahim, Professor für Kinderheilkunde und Direktor der Universitätskinderklinik in Jena, einen kurzen Brief an Dr. Gerhard Kloos, den Leiter der Thüringer Landesheilanstalten Stadtroda. Es ging um einen zwei Jahre alten Jungen, den Ibrahim vermutlich in der ambulanten Sprechstunde untersucht hatte. Das geistig behinderte Kind litt an schweren Krämpfen. Ibrahims Empfehlung: „Offenbar aussichtslose Zukunft. Vielleicht könnte er bei Ihnen eine nähere Beobachtung und Beurteilung finden. Euth.?" Tatsächlich wurde das Kind am 9. Februar 1944 in die von Kloos geleitete "Kinderfachabteilung“ aufgenommen. Was bedeutete das? Stadtroda war Teil eines Netzwerks von etwa dreißig „Kinderfachabteilungen“, in denen zwischen 1939 und 1945 zwischen 5.000 und 10.000 Säuglinge, Kinder und Jugendliche ermordet wurden. Auch der kleine Junge, den Ibrahim überwiesen hatte, überlebte die „Kinderfachabteilung " nicht. Ende Mai erkrankte er - den Eintragungen im Krankenblatt zufolge an einem fieberhaften Darminfekt. Das Kind starb am 2. Juni 1944, als Todesursache wurde „Herz-Kreislaufschwäche“ vermerkt. Es ist mehr als wahrscheinlich, dass bei seinem Tod "nachgeholfen“" wurde und sei es auch nur durch Unterlassen.

Es war nicht der erste und nicht der einzige Fall dieser Art. Zwischen 1941 und 1945 wurden nachweislich sieben Kinder aus der Jenaer Universitätskinderklinik nach Stadtroda überwiesen, die dort auch zu Tode kamen. In zwei Fällen liegen handschriftliche Überweisungen Ib- rahims vor, die offen „Euthanasie“ vorschlagen. 1943 hatte sich Dr. Herbert Linden, der als „Reichsbeauftragter für Heilund Pflegeanstalten“ eine zentrale Rolle im „Euthanasie“-Apparat spielte, beim Rektor der Universität Jena beschwert, dass die Jenaer Kinderklinik immer wieder Vermerke wie „Euthanasie beantragt“ oder „Die beantragte Euthanasie ist noch nicht bewilligt" in die Krankengeschichten eintrage. ${ }^{1}$ Das war heikel, weil die Kinder-„Euthanasie“ als geheime Reichssache eingestuft war und nicht an die Öffentlichkeit dringen sollte.

Es ist zu betonen, dass die Zuarbeit Ibrahims auffreiwilliger Basis erfolgte. Es gab keine gesetzliche Grundlage, die ihn dazu verpflichtet hätte. Den Entwurf zu einem „Gesetz über Sterbehilfe“, das der medizinische Expertenstab der „Euthanasie“-Aktion ausgearbeitet hatte, lehnte Hitler 1940

\footnotetext{
1 Renate Renner/Susanne Zimmermann, Prof. Dr. Jussuf Ibrahim und die NS-Kindereuthanasie, in: Ärzteblatt Thüringen 14 (2003), H. 7/8, S. 522525, 597-599 (Zitate: S. 522, 597); dies., Der Jenaer Kinderarzt Jussuf Ibrahim (1877-1953) und die Tötung behinderter Kinder während des Nationalsozialismus, in: Uwe Hoßfeld/Jürgen John/Oliver Lemuth/Rüdiger Stutz (Hg.), , ,Kämpferische Wissenschaft". Studien zur Universität Jena im Nationalsozialismus, Köln u.a. 2003, S. 437-451; Susanne Zimmermann, „Euthanasie wäre durchaus zu rechtfertigen ....". Der Jenaer Professor Jussuf Ibrahim und die NS-Kindermorde, in: Ralf Forsbach (Hg.), Medizin im „Dritten Reich". Humanexperimente, „Euthanasie“ und die Debatten der Gegenwart, Hamburg u.a. 2006, S. 81-98. Vgl. jetzt auch: Sandra Liebe, Prof. Dr. med. Jussuf Ibrahim (1877-1953). Leben und Werk, Diss. Universität Jena 2006.
}

ab. Er wollte, dass der Krankenmord - frei von allen bürokratischen Hindernissen und juristischen Einschränkungen - im rechtsfreien Raum stattfand. Die „Euthanasie“ blieb daher bis 1945 nach dem Gesetz in Deutschland strafbar. Kein Arzt konnte gezwungen werden, sich daran zu beteiligen, und die Organisatoren hüteten sich, Druck auf die Mediziner auszuüben. Sicher: Es bestand eine Meldepflicht für Kinderärzte, Geburtshelfer und Hebammen. Sie hatten behinderte Säuglinge und Kleinkinder den Gesundheitsämtern anzuzeigen, welche die Meldungen an die „Euthanasie“-Zentrale weiterleiteten. Die Meldepflicht wurde jedoch nicht allzu streng eingehalten, und die „Euthanasie“-Zentrale hatte wegen des konspirativen Charakters ihrer Organisationsstruktur auch kaum Zwangsmittel zur Hand, um die Meldepflicht durchzusetzen.

Aber Ibrahims Kontakte folgten ohnehin nicht dem bürokratischen Procedere: Er ergriff von sich aus die Initiative und nahm auf direktem Weg - von Kollege zu Kollege - Kontakt zu Kloos auf. Und er beschränkte sich nicht darauf, den Fall zu melden, er deutete von sich aus die Möglichkeit der „Euthanasie“ an - das wog schwer angesichts der Autorität Ibrahims auf dem Gebiet der Kinderheilkunde. Seine aktive Rolle innerhalb des Tatgeschehens ist hervorzuheben - und es handelte sich hier, wie die jüngere Forschung zeigt, keineswegs um einen Einzelfall.

Man muss sich klarmachen, dass die „Euthanasie" auf eine solche freiwillige Zuarbeit aus den Reihen der Ärzteschaft angewiesen war. Ein dichtes Netzwerk kollegia- 
ler Kontakte lieferte Hinweise auf Säuglinge, Kinder und Jugendliche, die dann in die "Kinderfachabteilungen" verlegt wurden. Ohne die mittelbare Beteiligung einer Vielzahl von Ärzten hätte der Vernichtungsapparat längst nicht so effektiv arbeiten können. Die Frage nach den Motiven für diesen vorauseilenden Gehorsam ist nicht leicht zu beantworten. Politischer Druck, ängstliche Anpassung, beruflicher Ehrgeiz, ideologische Indoktrinierung - das alles mag in dem einen oder anderen Fall eine Rolle gespielt haben. Entscheidend war es sicher nicht. Dazu sind die Quellen, die bei den an der NS-„Euthanasie“ mittelbar oder unmittelbar beteiligten Ärzten auf ein hohes Maß an Zustimmung, ja Begeisterung hindeuten, zu zahlreich. Handlungsleitend war in vielen Fällen eine regelrechte Aufbruchstimmung, das Hochgefühl, an einem sozialsanitären Großprojekt von welthistorischer Bedeutung beteiligt zu sein. Um dies verstehen zu können, müssen wir uns zunächst die besondere Signatur jenes Massenmordes an psychisch kranken und geistig behinderten Menschen vergegenwärtigen, den das nationalsozialistische Regime vor dem Hintergrund des Zweiten Weltkrieges ins Werk setzte.

Man kann den Nationalsozialismus mit guten Gründen als eine biopolitische Entwicklungsdiktatur auffassen, die darauf abzielte, die Kontrolle über Geburt und Tod, Sexualität und Fortpflanzung, Körper und Keimbahn, Variabilität und Evolution an sich zu bringen, den Genpool der Bevölkerung von allen unerwünschten „Beimischungen" $\mathrm{zu}$ „reinigen" und auf diese Weise einen homogenen "Volkskörper" zu schaffen. „Volkskörper" - das ist der Schlüsselbegriff nationalsozialistischer Biopolitik. Für Dr. Leonardo Conti etwa, der seit 1939 als „Reichsgesundheitsführer" an der Spitze der staatlichen wie auch der parteiamtlichen Gesundheitsführung stand, hatte der Staat für den Nationalsozialismus „nur Sinn als die Organisationsform des deutschen Volkes. Sinn und Zweck gibt ihm erst der lebendige Volkskörper". Der Nationalsozialismus habe den Begriff des Volkes „vertieft“. „Das Volk ist nicht die Summe der Staatsbürger des Landes, [...] sondern [...] die rassische und damit im Zusammenhang stehende kulturelle, geistige und seelische Gemeinschaft, deren äußerer Ausdruck erst die staatliche Verbundenheit ist."
Doch auch diese Begriffsbestimmung, so schränkte Conti ein, sei noch zu eng gefasst, „da sie nur die heutige, gegenwärtige Generation“ bezeichne. Der Begriff „Volk“ umfasse aber auch die dahingegangenen und vor allem die kommenden Generationen. Daraus leitete Conti als Aufgabe des Staates $\mathrm{ab}$, die Rahmenbedingungen dafür zu schaffen, dass sich das Volk „von Generation zu Generation kraftvoller und schöner erneuern“ könne. Diese Staatsaufgabe zerfalle in zwei Gebiete, die „Erbgesundheitspflege“ und die „Anlageförderung“. Die eine forme das „Erbbild“, die andere das „Erscheinungsbild des Volksgenossen“.

Es war von vornherein klar, dass innerhalb einer solchen biopolitischen Entwicklungsdiktatur der Medizin eine Schlüsselstellung zugewiesen würde - und dass sich der Kinderheilkunde im „Dritten Reich“ ganz neue Perspektiven eröffneten: etwa auf dem Feld der Vorbeugung, der systematischen Reihenuntersuchungen von Kindern und Jugendlichen, der Gesundheitserziehung und Gesundheitsführung der Jugend, der Körperertüchtigung, des Kampfes gegen Alkohol und Nikotin, der Sexualhygiene und des Kampfes gegen Geschlechtskrankheiten. Das bedeutete - über die traditionellen Tätigkeitsbereiche der Säuglingsund Kleinkinderfürsorge, der Behandlung von Infektionskrankheiten und Ernährungsstörungen hinaus - eine Ausweitung der Kinderheilkunde auf neue Praxis- und Forschungsfelder, die Erweiterung der pädiatrischen Zuständigkeit auf Jugendliche bis zur Geschlechtsreife und eine Expansion des Kompetenzbereichs der Pädiater gegenüber den Schulärzten und den Ärzten des Gesundheitsdienstes der Hitlerjugend. Diese Perspektive war für die Pädiater und die Pädiatrie eine Verführung, zumal sie sich mit eigenen Zielvorstellungen traf. War doch die deutsche Kinderheilkunde seit den Anfängen ihrer Etablierung als eigenständige Fachdisziplin in der zweiten Hälfte des 19. Jahrhunderts mit dem Anspruch aufgetreten, durch die Senkung der Säuglings- und Kindersterblichkeit einen Beitrag zur Stärkung des Volkes und des Staates zu leisten, die zu ihrem Fort-

\footnotetext{
2 Leonardo Conti, Körperliche Ertüchtigung als biologische Aufgabe des Staates, Typoskript, Teilnachlass Leonardo Conti, Privatbesitz (demnächst Bundesarchiv Berlin).
}

bestand auf eine zahlreiche, gesunde und leistungsstarke Nachkommenschaft angewiesen seien. Und der Bedeutungszuwachs der deutschen Kinderheilkunde nach dem verlorenen Ersten Weltkrieg, die Anerkennung als Spezialdisziplin mit einer dreijährigen Ausbildungszeit Mitte der 1920er Jahre, hing eng mit der Erwartung zusammen, die Kinderheilkunde werde die „Wiederaufforstung des deutschen Volksbestandes $^{\text {" }}{ }^{3}$ beschleunigt vorantreiben. Das war ein Pfund, mit dem die Pädiatrie auch nach 1933 wuchern konnte. Dass sich nun die Gewichte zunehmend von der kurativen zur präventiven Medizin, vom kranken zum gesunden Kind verschoben, wurde dabei als Chance, nicht als Risiko begriffen. Kinderheilkunde sollte um eine "Jugendmedizin“ erweitert und zur ,ärztlichen Jugendkunde" fortentwickelt werden. Dr. Wilfried Zeller, Schularzt in Berlin-Tiergarten, beschrieb 1936 das erweiterte Aufgabenspektrum des künftigen "Jugendarztes" mit den beiden Begriffen "Gesundheitspflege“ und „Gesundheitsführung“ der Jugend: „Gesundheitspflege bedeutet: Bewahrung vor Krankheit und körperlichem Schaden, Gesundheitsführung dagegen: Steigerung der Gesundheit zu höheren Stufen des Gesundseins. Denn wie in dem Begriff, krank' alle möglichen Steigerungsgrade des Krankseins ausgedrückt werden können, so umfasst der Begriff, gesund' neben der farblosen Bedeutung, nicht krank' alle Stufen immer besserer Gesundheit, das heißt, gesteigerter Widerstands- und Leistungsfähig-


den man immer enger und damit das eigene Arbeitsgebiet immer weiter fassen konnte dies schien der Pädiatrie immer neue Felder der Prophylaxe, der Sozialhygiene, der Gesundheitserziehung zu eröffnen.

Die Risiken dieses Ansatzes wurden nicht erkannt - oder aber billigend in Kauf genommen. Denn der Fokus ärztlichen Handelns verschob sich in der biopolitischen Entwicklungsdiktatur des Nationalsozialismus vom individuellen zum „Volkswohl“. Der Wert des Menschen leitete sich aus seiner Position und Funktion

\footnotetext{
3 Zit. n. Thomas Beddies, ,'Du hast die Pflicht, gesund zu sein“. Der Gesundheitsdienst der Hitler-Jugend 1933-1945, Berlin 2010, S. 67. 4 Wilfried Zeller, Der Jugendarzt, seine Aufgaben und Methoden, in: Gesundheit und Erziehung 49 (1936), S. 129-136, hier: S. 129.
} 


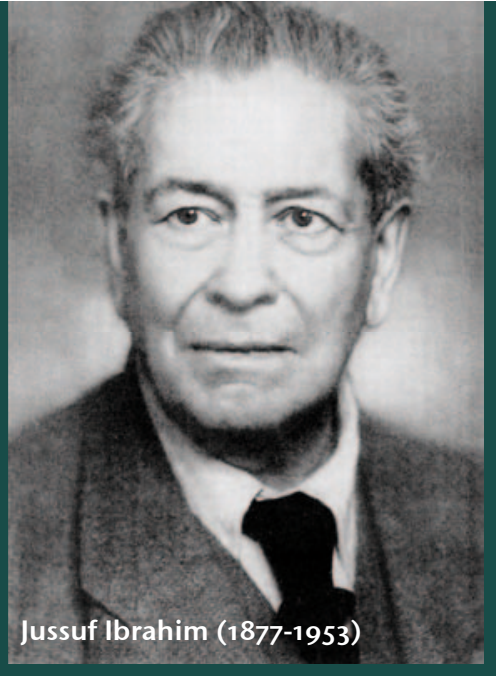

Sbür. Randesbeilanitalten Stadtroda

Xm II. 2. $19+3^{3}$.

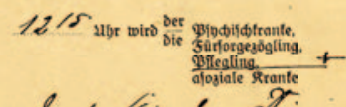

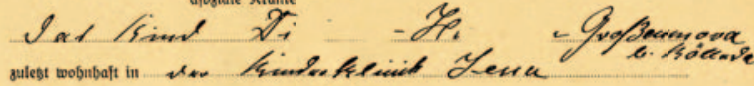

anis der Dem verforem



Den biefigen Infletten augetübrt. radridntib: Moar

In gapieren wurঠen abgegeben:

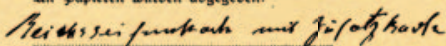

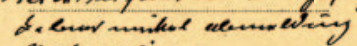

profore cimen

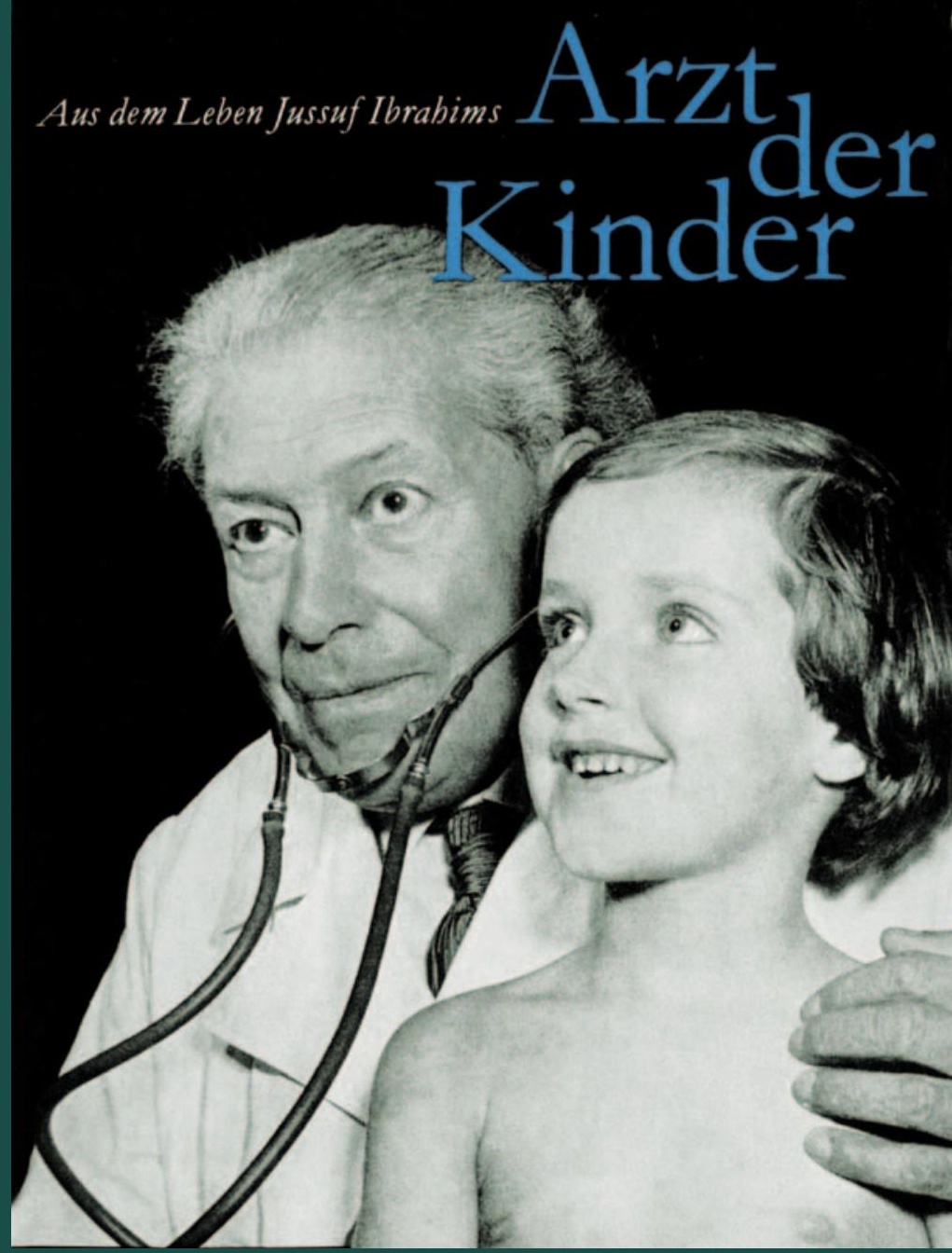

\section{Jena}

In den 1990er Jahren wurden Vorwürfe laut, der ehemalige Leiter der Jenaer Universitäts-Kinderklinik Jussuf Ibrahim sei in die Krankenmorde verwickelt gewesen, indem er Kinder aus seiner Klinik an die zuständige „Kinderfachabteilung“ des Landeskrankenhauses in Stadtroda selbst überwiesen bzw. dies geduldet habe. Eine Kommission der Universität Jena kam im April 2000 zu dem Ergebnis, dass aus der Kinderklinik zwischen 1941 und 1945 tatsächlich „insgesamt sieben schwerstgeschädigte Kinder nach Stadtroda überwiesen [wurden], die auch dort verstarben“, zudem liegen für zwei Kinder „handschriftliche Überweisungsschreiben Ibrahims vor, die offen ,Euthanasie' vorschlagen“.
Für den unbedarften Umgang mit den Krankentötungen durch Ibrahim sind Beschwerden des Direktors der Anstalt in Stadtroda, Gerhard Kloos, bezeichnend, wonach in der Kinderklinik die Frage der "Euthanasie“ offen mit den Eltern besprochen worden sei, obwohl doch, wie es aus dem Innenministerium hieß, nach außen hin die Tatsache, dass "in Einzelfällen Euthanasie gewährt werden kann, nicht in Erscheinung treten" sollte.

Einer Empfehlung der Kommission folgend wurde die seit 1953 nach Ibrahim benannte Kinderklinik 2001 in "Klinik für Kinder- und Jugendmedizin" umbenannt. 
in dem als Organismus höherer Ordnung verstandenen „Volkskörper“ ab - und für den „Volkskörper“ hatte ein Mensch nur dann einen Wert, wenn er "erbgesund“ und „rasserein“, leistungsstark und lebenstüchtig, fruchtbar und wehrhaft war.

Nun sah sich die Kinderheilkunde seit den Anfängen der Eugenik und Rassenhygiene am Ende des 19. Jahrhunderts dem Vorwurf ausgesetzt, sie setze mit ihrem Kampf gegen die Säuglings- und Kindersterblichkeit die „natürliche Auslese“ ein Stück weit außer Kraft und trage auf diese Weise zur Degeneration der Erbmasse des Volkes bei. Mit der Machtübernahme der Nationalsozialisten wurde nun die Rassenhygiene zur Staatsdoktrin erhoben, und die Kinderheilkunde musste sich dieser Herausforderung stellen. Die „Deutsche Gesellschaft für Kinderheilkunde“ - sie hatte sich mittlerweile im Zuge der „Selbstgleichschaltung" ihrer jüdischen und politisch missliebigen Mitglieder entledigt - tat dies auf ihrer Jahrestagung 1934. Nachdem die Versammlung sich von Prof. Otmar Frhr. v. Verschuer, Abteilungsleiter des Kaiser-Wilhelm-Instituts für Anthropologie, menschliche Erblehre und Eugenik in Berlin, dem damals führenden deutschen Humangenetiker, über den Stand der Erblehre des Menschen hatte unterrichten lassen, referierte Thilo Brehme über die „Aufgaben und Bedeutung der Kinderheilkunde im Neuen Deutschland“. Dabei setzte er sich auch mit „Vorwürfe[n] gegenüber der Pädiatrie wegen der Erhaltung lebensunwerten Lebens “ auseinander und wagte eine Prognose: „Es ist möglich, dass eine neue, mehr die Gesamtheit als das Einzelindividuum berücksichtigende ethische Grundhaltung uns einmal zwar nicht die Verpflichtung auferlegt, wirklich lebensunwertes Leben auszumerzen, aber vielleicht doch die Freiheit lässt, gegebenenfalls nichts zu seiner Erhaltung zu tun."

An diesem Punkt beginnt sich das Bild zu klären. Wir bekommen eine Vorstellung davon, warum so viele Pädiater - wie auch Kinder- und Jugendpsychiater - die Ermordung von bis zu 10.000 behinderten und kranken Kindern und Ju-

5 Thilo Brehme, Aufgaben und Bedeutung der Kinderheilkunde im neuen Deutschland, in: Monatsschrift für Kinderheilkunde 62 (1934), S. 183 ff., hier: S. 184. gendlichen in den „Kinderfachabteilungen " und weiterer 4.200 in den Gaskammern der "Aktion $\mathrm{T}_{4}$ “ widerspruchslos hinnahmen, warum manche - so wie Jussuf Ibrahim - die Gelegenheit nutzten, Kinder, bei denen keine Behandlungsperspektive bestand, in Einrichtungen der „Euthanasie“ abzuschieben, warum andere - wie der Direktor der UniversitätsKinderklinik in Leipzig, Prof. Werner Catel - im eigenen Haus eine „Kinderfachabteilung" einrichteten oder Kinder, die als „lebensunwert“ ermordet werden sollten, im Zuge „verbrauchender Forschung“ benutzten - so etwa Prof. Georg Bessau, der Direktor der Kinderklinik an der Charité, der in mehreren Testreihen Tuberkuloseimpfstoffe an solchen Kindern prüfen ließ. Es deutet sich an, warum Ärzte aktiv an diesem Massenmord teilnehmen konnten, ohne darin einen Widerspruch zum eigenen ärztlichen Selbstverständnis zu sehen. Wie etwa der Berliner Kinderarzt Dr. Ernst Wentzler, eine der Schlüsselfiguren der Kinder-,Euthanasie“, der 1942 - die Mordaktion lief auf vollen Touren - den Vorsitz des neu gegründeten Vereins „Deutsches Kinderkrankenhaus e.V.“ übernahm - eines Vereins, der sich den Bau mustergültiger Kinderkrankenhäuser zum Ziel gesetzt hatte. Die Dialektik von Heilen und Vernichten ermöglichte es allen diesen Medizinern, sich als „Arzt am Volkskörper“ zu verstehen - und die schwächsten unter ihren kleinen Patienten preiszugeben.

Es ist wichtig, diesen Motivlagen immer wieder nachzuspüren - auch mit Blick auf die Gegenwart, auf aktuelle Diskussionen um Präimplantationsdiagnostik und Designerbabys. Allen neo-eugenischen Sozialutopien müssen wir das skeptische Wort Immanuel Kants entgegensetzen: „Aus so krummem Holze, als woraus der Mensch gemacht ist, kann nichts ganz Gerades gezimmert werden." ${ }^{6}$ Behinderung, Krankheit, Schwäche und Hilflosigkeit gehören - wie Empathie, Respekt, Demut, Erbarmen und Güte - zur conditio humana. Hier kommt gerade auch der Medizin eine große Verantwortung

\footnotetext{
${ }^{6}$ Immanuel Kant, Idee zu einer allgemeinen Geschichte in weltbürgerlicher Absicht, in: ders., Werke in 6 Bänden, Bd. 6, hg. v. Wilhelm Weischedel, Darmstadt 1975, S. 33-50, hier: S. 41.
}

$\mathrm{zu}$ - eine Verantwortung, die auch Grenzen medizinischer Praxis und medizinischer Wissenschaft respektiert, die kompromisslos den einzelnen Menschen zum Ausgangspunkt der Berufsethik macht. Das heißt vielleicht auch: Erwartungen enttäuschen, Zumutungen zurückweisen, Druck standhalten. Am Ende gilt: Ein Gemeinwesen ist dann am stärksten, wenn es vom Schwächsten her denkt.

\section{Korrespondenzadresse}

apl. Prof. Dr. Hans-Walter Schmuhl

Fakultät für Geschichtswissenschaft, Philosophie und Theologie

der Universität Bielefeld

Storchsbrede 2

33613 Bielefeld

schmuhl@schmuhl-winkler.de 
„Im Gedenken der Kinder. Kinderärzte und die Verbrechen an Kindern in der NS-Zeit“ lautet der Titel einer die DGKJ-Jahrestagung in Potsdam-Babelsberg begleitenden Ausstellung, die über die Einbeziehung kranker und behinderter Kinder in die Medizinverbrechen des Nationalsozialismus und die Verwicklung der Kinderärzte in diese Taten informiert. Die Ausstellung wurde in Zusammenarbeit mit der Brandenburgischen Historischen Kommission realisiert und belegt umfassend den medizinischen und ordnungspolitischen Zugriff auf die minderjährigen Opfer der NS-Medizin. Sie wird Anfang 2011 auch im Haus der Brandenburgisch-Preußischen Geschichte in Potsdam gezeigt; weitere Präsentationen sind u.a. in Leipzig und Berlin geplant.

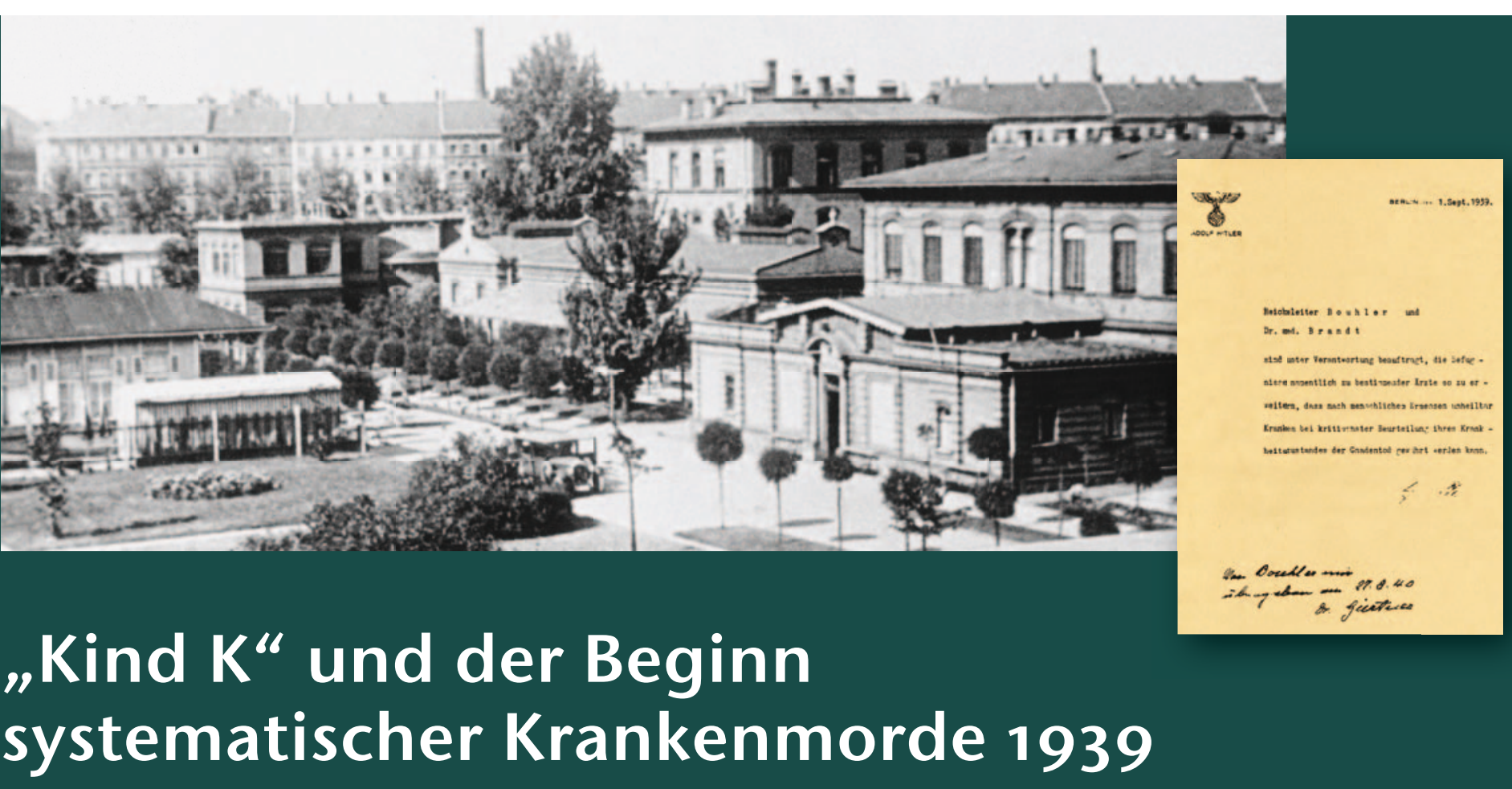

Im Frühjahr 1939 richtete ein Elternpaar aus Sachsen ein Gesuch zur Tötung seines schwer behinderten Säuglings an Adolf Hitler. Das Schreiben wurde in Hitlers Privatkanzlei, der "Kanzlei des Führers der NSDAP“, bearbeitet. Ihr Leiter, Philipp Bouhler, und Hitlers Begleitarzt, der Chirurg Karl Brandt, wurden von Hitler mit der Prüfung des Falls „Kind K“ beauftragt. Karl Brandt überbrachte dem Direktor der Universitäts-Kinderklinik Leipzig, Werner Catel, persönlich die Zustimmung Hitlers zur "Einschläferung" des Kindes. Wahrscheinlich Ende Juli 1939 wurde es in der Klinik getötet.

Der Fall des "Kindes K" soll dazu beigetragen haben, dass Hitler im Herbst 1939 Brandt und Bouhler mit der Durchführung eines umfassenden „Euthanasie“-Programms beauftragte. Das entsprechende Schriftstück wurde auf den 1. September 1939, den Tag des Kriegsbeginns, datiert; mit der Aggression nach außen begann damit auch die „Vernichtung lebensunwerten Lebens" im eigenen Land. Die Krankenmorde unterlagen strikter Geheimhaltung; ein "Euthanasie“-Gesetz wurde nie verabschiedet.

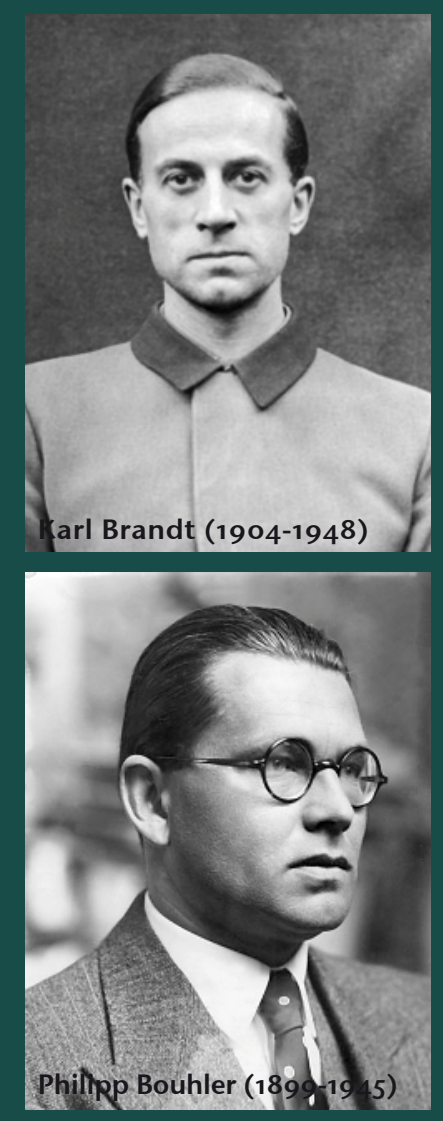




\section{Das „Reichsausschuß“-Verfahren}


2uszug aus Dem RDErl. Des Reidsminifters Des Jnnern vom 18.8. 1939 - IVb 3088/39-1079 Mi -, betr. शneldepflidt für mißßgeftaltete u[w. Neugeborene

Die "Kinder-Euthanasie" oblag dem Amt Ilb der Kanzlei des Führers. Unter der irreführenden Bezeichnung „Reichsausschuß zur wissenschaftlichen Erfassung erb- und anlagebedingter schwerer Leiden“ regelten zwei Sachbearbeiter, der Diplomlandwirt Dr. Hans Hefelmann und der Bankkaufmann Richard von Hegener, die Meldung, Begutachtung, Einweisung und schließlich Tötung von mehr als 5000 Kindern. Organisatorisch wurden sie von der Gesundheitsabteilung des Reichsinnenministeriums (Dr. med. Herbert Linden) unterstützt; bereits am 18. August 1939 war ein vertraulicher Erlass zur Meldung behinderter Kleinkinder herausgegeben worden. Die zunächst wohl noch in gutem Glauben von Hebammen und Ärzten ausgefüllten Meldebogen dienten drei „Fachgutachtern“ als Grundlage zur Selektion "lebensunwerter" Kinder.
Gutachter im „Reichsausschuß“-Verfahren waren die Kinderärzte Werner Catel aus Leipzig und Ernst Wentzler aus Berlin sowie der Psychiater Hans Heinze aus Brandenburg-Görden. Befürworteten sie die Tötung eines Kindes, so wurden die Eltern aufgefordert, ihre Tochter oder ihren Sohn in eine von rund 30 „Kinderfachabteilungen" aufnehmen zu lassen, die zumeist pädiatrischen oder psychiatrischen Kliniken angeschlossen waren. Dort wurden die Kinder von den zuständigen Ärztinnen und Ärzten in der Regel mit Medikamenten vergiftet. Nicht selten ging der Tötung eine Zeit der klinischen Beobachtung voraus; in etwa der Hälfte der Einrichtungen fand begleitende Forschung statt. 


\section{Gutachter im „Reichsausschuß zur wissenschaftlichen Erfassung von erb- und anlagebedingten schweren Leiden“}

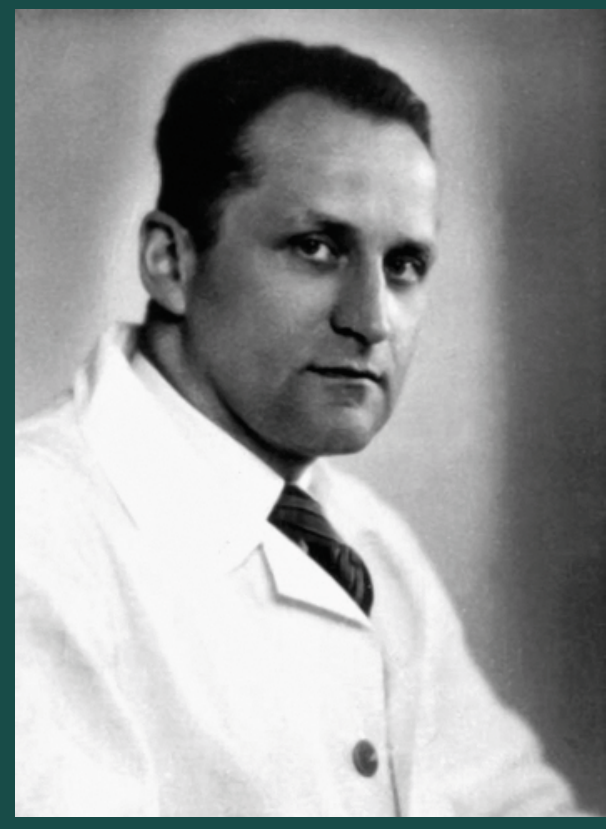

Werner Catel (1894-1981)

Direktor der Universitäts-Kinderklinik Leipzig; über den Fall "Kind $K^{\prime \prime}$ von Beginn an in das "Reichsausschuß"-Verfahren eingebunden. Nach 1945 zunächst in Hessen ärztlich tätig, war Catel 1954 bis 1962 Professor für Kinderheilkunde an der Universität Kiel. Er rechtfertigte die "Euthanasie“ kranker und behinderter Kinder nach 1945 in zahlreichen Publikationen unter Leugnung jeder individuellen Schuld im Hinblick auf die Medizinverbrechen in der NS-Zeit.

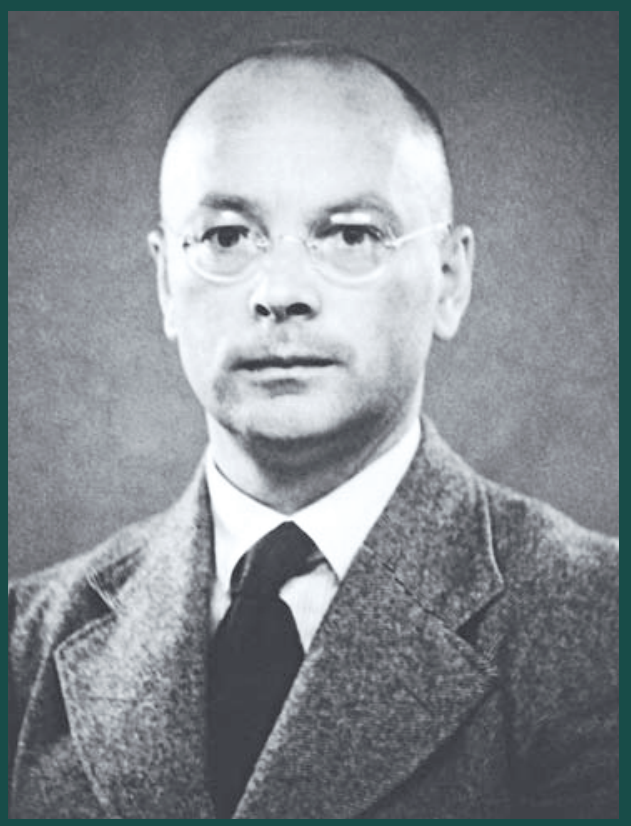

Hans Heinze (1895-1983)

Psychiater mit kinder- und jugendpsychiatrischer Spezialisierung; seit 1934 im brandenburgischen Anstaltsdienst, seit 1938 Leiter der Anstalt Brandenburg-Görden mit rund 1000 Betten. Heinze arbeitete u.a. eng mit dem Kaiser-WilhelmInstitut für Hirnforschung in Berlin-Buch (Julius Hallervorden) zusammen. Kurz nach Entlassung aus russischer Haft wurde er 1954 Leiter der jugendpsychiatrischen Klinik in Hannover-Wunstorf.

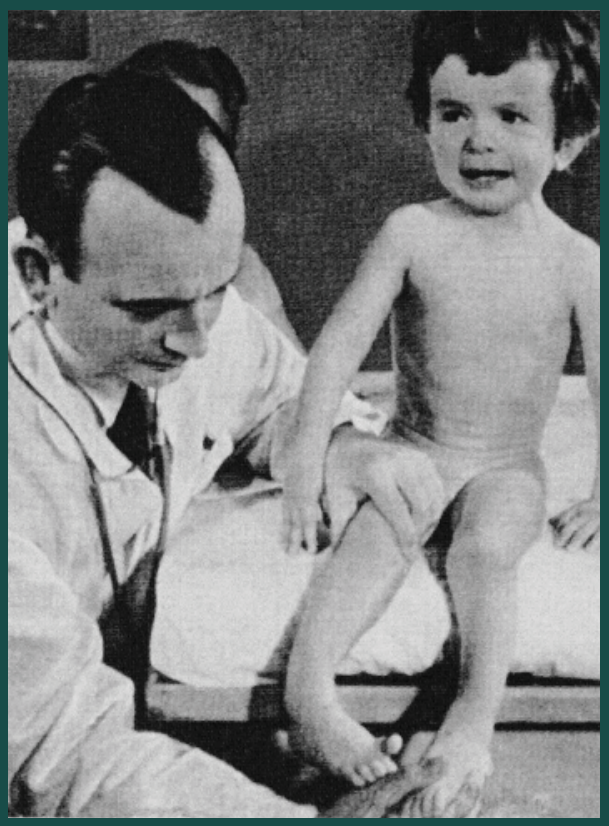

\section{Ernst Wentzler (1891-1973)}

Besitzer einer privaten Kinderklinik in Berlin-Frohnau; verfügte über enge persönliche Kontakte zur Kanzlei des Führers. Wentzler gehörte zum engsten Kreis der ärztlichen Berater und Organisatoren der Krankenmordaktionen; in seiner Privatklinik gab es keine Kinderfachabteilung.

Nach 1945 war er als niedergelassener Kinderarzt in Hannoversch Münden tätig. 


\section{Karte der „Kinderfachabteilungen“}

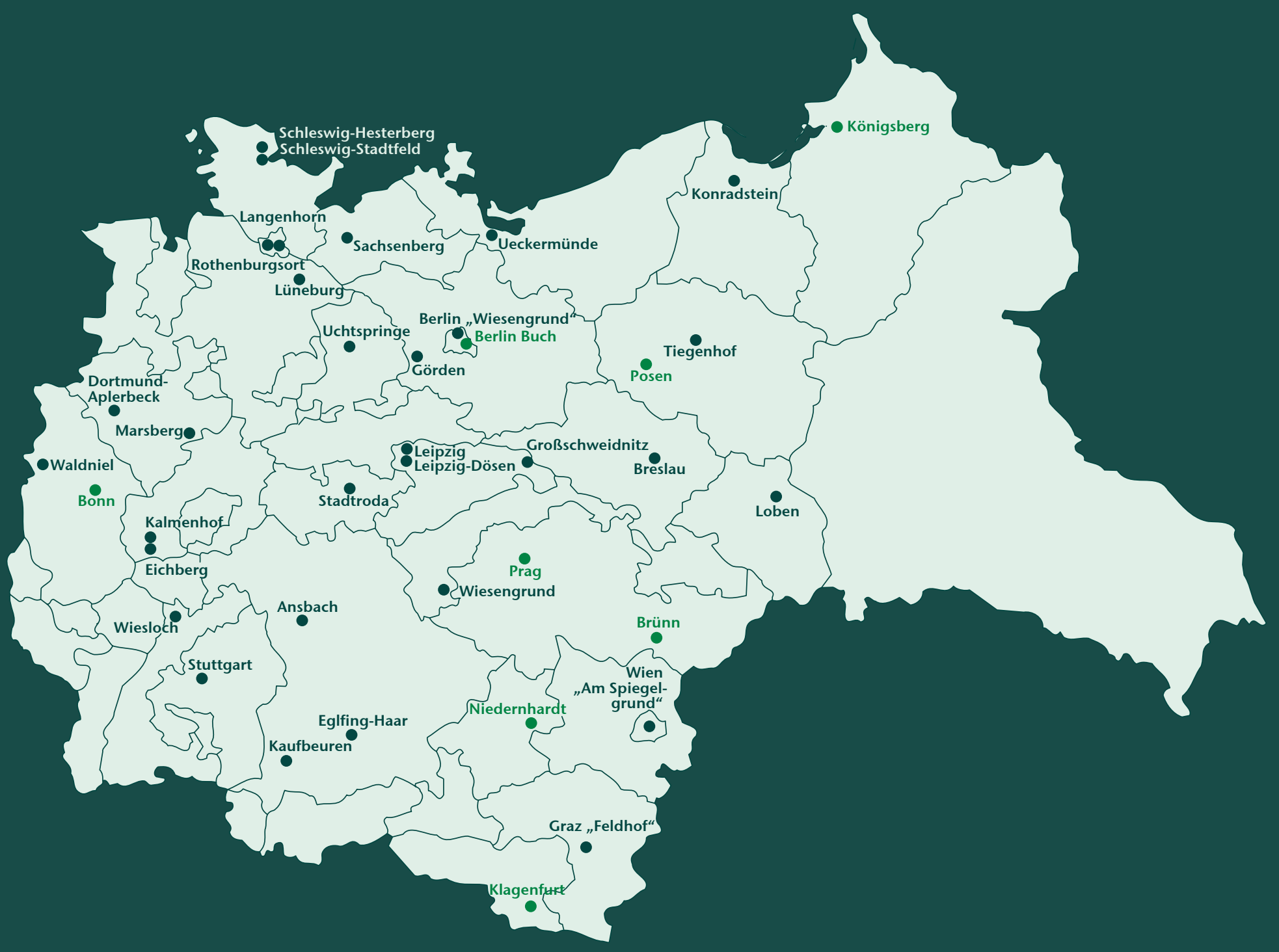

- Gesicherter Standort

- Noch nicht gesicherter Standort 

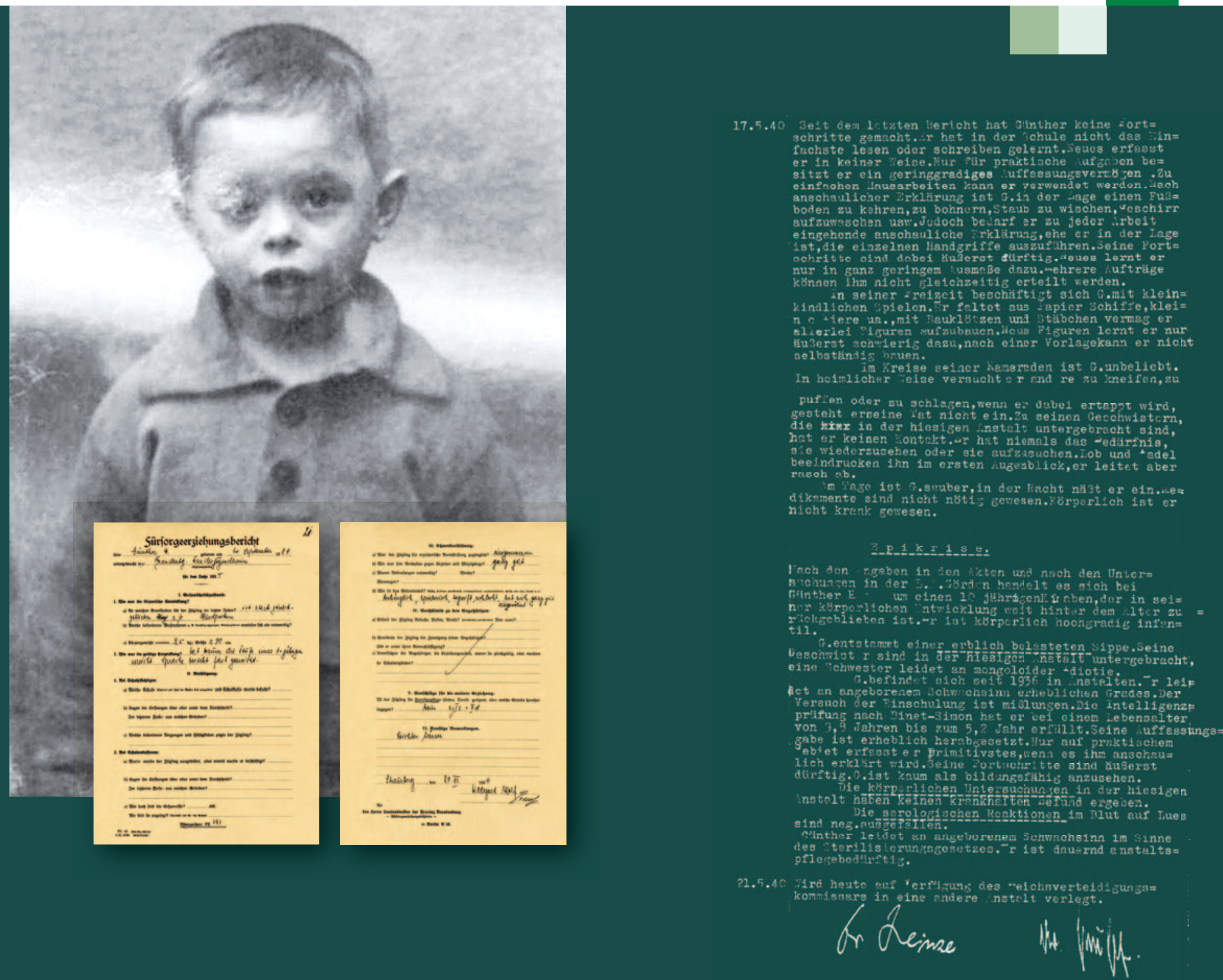

\section{Günther E. - „Wie anhänglich er ist, beweist er jeden Tag"}

Der 1929 geborene Günther stammte aus einer Arbeiterfamilie in Wittstock/Dosse. Wegen "Verwahrlosung" kamen er und seine drei Schwestern 1934 zunächst in Fürsorgeerziehung; zwei Jahre später wurden die Geschwister „zur geistigen und körperlichen Förderung" in die Landesanstalt Potsdam verlegt. Mit der Auflösung der Anstalt 1938 gelangten die vier Kinder nach Brandenburg-Görden. Günther wurde den „bildungsunfähigen“ Kindern zugerechnet: „nicht beschulungsfähig“, heißt es im Meldebogen, auch Besuch erhalte er "keinen“. Am 21. Mai 1940 wurde der 10-jährige Junge in der Gaskammer des benachbarten Brandenburger Zuchthauses ermordet.

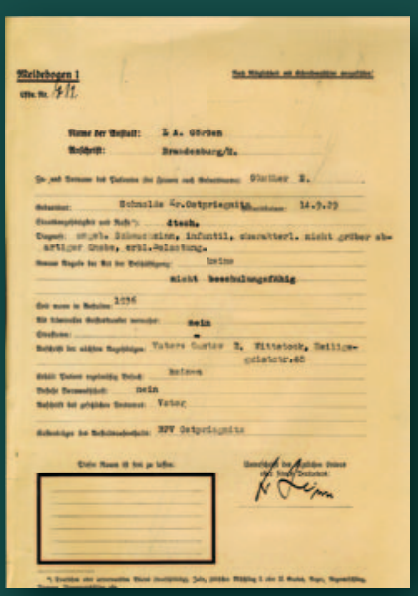


Brief einer Mutter an Prof. Duken

Sehr verehrter Herr Professor !

...

Unsere liebe kleine Christel ist nach 5tägigem Aufenthalt in der Heilanstalt Eichberg am 30. Juni gestorben. Ich war sehr erschrocken über diesen schnellen Tod in Eichberg und glaubte zuerst das Kind sei dort doch nicht gut versorgt worden, da ich es noch so munter von Heidelberg dorthin brachte. Wenn ich geahnt hätte, daß das kI. Leben sowieso bald erlöschen würde, hätte ich diese beschwerliche Reise nicht mehr unternommen und hätte das


Kind noch in Heidelberg gelassen. Hatten Sie es damals schon für möglich gehalten, daß das Kind so schnell sterben würde? Ich denke an Ihre Worte, daß das Kind wohl doch nicht alt geworden wäre, und auch nimmer gesund geworden wäre, und tröste mich mit dem Gedanken, daß es erlöst ist, von seinem schweren Leiden. Uns Eltern ist damit eine grosse Sorge für die Zukunft genommen. Das Kind wurde in Eichberg sektiert. ... Wir Eltern würden natürlich auch gerne näheres darüber erfahren. Da ich den Arzt der Anstalt ja kaum kenne, würde ich Sie bitten, sich den Bescheid von dort geben zu lassen und mir nochmal zu schreiben, wenn es Ihre Zeit nicht übermässig beansprucht. Ich habe in den Tagen, in denen Christel bei Ihnen in der Klinik war, grosses Vertrauen zu lhnen gefasst und komme deshalb auch heute nochmal zu Ihnen mit meiner Bitte....

Ich danke Ihnen sehr herzlich und grüsse Sie bestens Ihre Mathilde N.

\section{Brief von Prof. Duken an einen Vater}

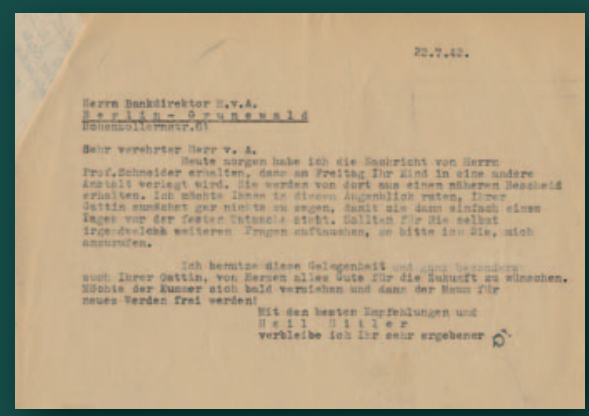

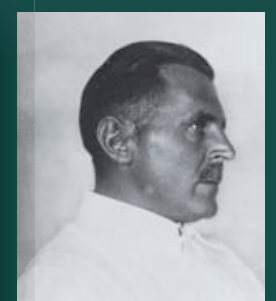

Johann Duken (1889-1954)

\section{Heidelberg}

In Heidelberg bestand keine „Kinderfachabteilung“; Kinder, die aus der Universitätskinderklinik unter Johann Duken dem „Reichsausschuß“ gemeldet wurden, verlegte man - nicht selten über die Psychiatrische Universitätsklinik unter Carl Schneider - in die nahegelegene Anstalt Eichberg (Dr. Friedrich Mennecke), wo sie getötet wurden.

Aber auch unabhängig von dem „Reichsausschuß“-Verfahren im engeren Sinne wurden in der Kinderklinik Patienten durch Nichtbehandlung und Nahrungsentzug getötet: „Da es sich um ein minderwertiges Kind handelt, wird keine Frauenmilch gegeben“, heißt es in einer Akte, und in einem weiteren Fall eines "minderwertigen“ Kindes mochte die behandelnde Ärztin "nur hoffen, dass es bald ad exitum kommt. “ Dukens Einstellung war dabei in Heidelberg und sogar bei den alliierten Kriegsgegnern durchaus bekannt; nicht zuletzt in seinen Vorlesungen soll er die „unbemerkte Euthanasierung“ geistig behinderter Kinder offensiv vertreten haben. 
Diktat in der Intelligenzprüfung (1942):

"Schwer war der Winter für das Volk, noch schwerer draußen für unsere Truppen. Das Deutsche Volk aber wußte, daß es alles auf sich nehmen mußte, um die Voraussetzungen für den Sieg draußen an der Front zu schaffen. Die Haltung unseres Volkes im Innern war deshalb der Haltung unserer Kämpfer draußen ebenbürtig. Beide, die Front in der Heimat, vor allem aber die Front draußen, haben soeben die Anerkennung und den Dank des Führers in einer Weise gesagt bekommen, wie sie schöner und größer nicht ausgesprochen sein kann. (...)“

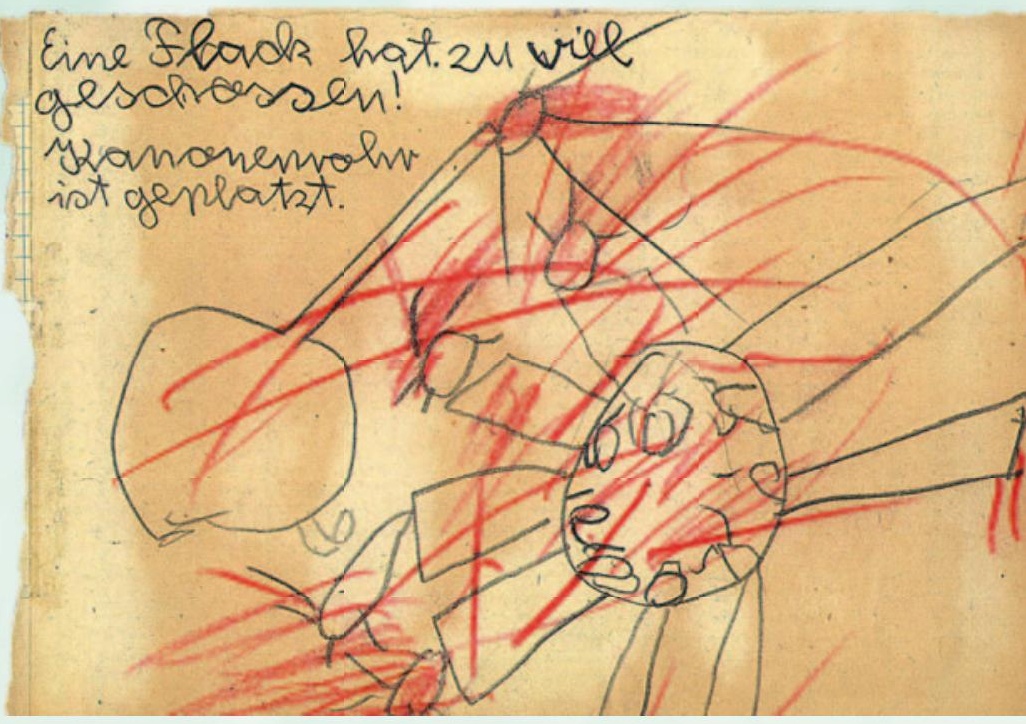




\section{Organ der Deutschen Gesellschaft für Kinder- und Jugendmedizin (DGKJ) Organ der Österreichischen Gesellschaft für Kinder- und Jugendheilkunde (ÖGKJ)}

\author{
Federführende Schriftleiter / Editors-in-chief \\ Prof. Dr. M.J. Lentze, Abteilung für allgemeine Pädiatrie und Poliklinik, \\ Zentrum für Kinderheilkunde der Universität Bonn \\ Prof. Dr. F. Zepp, Zentrum für Kinder- und Jugendmedizin, \\ Universitätsmedizin, Johannes-Gutenberg-Universität Mainz \\ Prof. Dr. R. Kerbl, LKH Leoben-Eisenerz, Abteilung für Kinder- \\ und Jugendliche
}

\section{Rubrikherausgeber / Section editors \\ Leitthema / Main topic (Review articles) \\ Prof. Dr. M.J. Lentze, Bonn - Prof. Dr. F. Zepp, Mainz \\ Prof. Dr. R. Kerbl, Leoben}

\section{Originalien (Kasuistik, Klinik und Forschung)/ Original articles (Case reports, clinic and research) Prof. Dr. B. Koletzko, München • Prof. Dr. S. Wirth, Wuppertal Prof. Dr. F. Zepp, Mainz}

\section{Konsensuspapiere / Consensus papers \\ Prof. Dr. S. Wirth, Wuppertal}

Bild des Monats / Image of the month PD Dr. R. Beetz, Mainz • PD Dr. L. Weber, München

\section{Arzneimitteltherapie / Drug therapy}

Prof. Dr. Dr. h.c. W. Rascher, Erlangen - Prof. Dr. H.W. Seyberth, Landau

\section{CME Weiterbildung • Zertifizierte Fortbildung / Continuing medical education \\ Prof. Dr. B. Koletzko, München • Prof. Dr. W. Sperl, Salzburg}

\section{Pädiatrie aktuell / Trends in pediatrics}

Univ. Doz Dr. Dr. h.c. O. Bodamer, Miami • Prof. Dr. J. Freihorst, Aalen Prof. Dr. R. Kerbl, Leoben • Dr. G. Krandick, Oberhaching

\author{
Leserforum / Reader's forum \\ Prof. Dr. M.J. Lentze, Bonn • Prof. Dr. F. Zepp, Mainz \\ Prof. Dr. R. Kerbl, Leoben
}

\author{
Mitteilungen der DGKJ und ÖGKJ / \\ News of the societies \\ Prof. Dr. M. Weiß, Köln (DGKJ) • Prof. Dr. R. Kerbl, Leoben (ÖGKJ)
}

\section{Präsident der DGKJ \\ Prof. Dr. F. Zepp, Mainz \\ Präsident der ÖGKJ \\ Prof. Dr. K. Schmitt, Linz}

\begin{abstract}
Wissenschaftlicher Beirat / Advisory board Prof. Dr. H. Böhles, Frankfurt • Prof. Dr. G.F. Bottazzo, Rom Prof. Dr. K.-M. Debatin, Ulm • Prof. Dr. J. H. H. Ehrich, Hannover Prof. Dr. M. Gahr, Dresden • Prof. Dr. J. Gärtner, Göttingen Prof. Dr. A. Grüters, Berlin • Prof. Dr. G. Hansen, Hannover Prof. Dr. E. Harms, Münster • Prof. Dr. J. Hess, München Prof. Dr. G. F. Hoffmann, Heidelberg • Prof. Dr. H.-I. Huppertz, Bremen Prof. Dr. W. Kiess, Leipzig • Prof. Dr. I. Krägeloh-Mann, Tübingen Prof. Dr. H.-H. Kramer, Kiel • Prof. Dr. E. Mayatepek, Düsseldorf Prof. Dr. W. Müller, Graz • Prof. Dr. C. Niemeyer, Freiburg Prof. Dr. F. Riedel, Hamburg • Prof. Dr. R. Seger, Zürich Prof. Dr. F. H. Sennhauser, Zürich • Prof. Dr. G. Simbruner, Innsbruck Prof. Dr. C.P. Speer, Würzburg • Prof. Dr. K. Ullrich, Hamburg Prof. Dr. D. von Schweinitz, München • Prof. Dr. N. Wagner, Aachen Prof. Dr. U. Wahn, Berlin • Prof. Dr. M. Weiß, Köln • Prof. Dr. K.-P. Zimmer, Gießen
\end{abstract}

\section{Begründet 1903 und herausgegeben von}

Prof. Dr. A. Keller • Prof. Dr. A. Czerny • Prof. Dr. G. Bessau Prof. Dr. H. Kleinschmidt

\section{Aims \& Scope}

Monatsschrift Kinderheilkunde is an internationally respected journal covering all areas of pediatrics and juvenile medicine. The focus is on prevention, diagnostic approaches, management of complications, and current therapy strategies. The journal provides information for all pediatricians working in practical and clinical environments and scientists who are particularly interested in issues of pediatrics.

Freely submitted original papers allow the presentation of important clinical studies and serve scientific exchange.

Comprehensive reviews on a specific topical issue focus on providing evidenced based information on diagnostics and therapy.

Review articles under the rubric "Continuing Medical Education" present verified results of scientific research and their integration into daily practice.

Review: All articles of Monatsschrift Kinderheilkunde are reviewed. Original papers and case reports undergo a peer review process.

Declaration of Helsinki: All manuscripts submitted for publication presenting results from studies on probands or patients must comply with the Declaration of Helsinki. Indexed in Science Citation Index Expanded, EMBASE and Scopus. 